\title{
Avaliação eletromiográfica dos músculos da mastigação de indivíduos com desordem temporomandibular submetidos a acupuntura
}

\author{
Surface electromyography of masticatory muscles in individuals with \\ temporomandibular disorder submitted to acupuncture
}

\author{
Graciele da Silva Borin ${ }^{1}$, Eliane Castilhos Rodrigues Corrêa ${ }^{2}$, Ana Maria Toniolo da Silva ${ }^{3}$, \\ Jovana de Moura Milanesi ${ }^{1}$
}

\begin{abstract}
RESUMO
Objetivo: Avaliar o efeito da acupuntura na atividade eletromiográfica dos músculos mastigatórios em pacientes com desordem temporomandibular (DTM). Métodos: Quarenta mulheres, de 20 a 40 anos e com diagnóstico de DTM foram avaliadas por meio da eletromiografia dos músculos masseter e temporal (fascículo anterior). As participantes foram distribuídas em grupos de estudo (GE), cujo tratamento com acupuntura foi aplicado logo após a avaliação inicial, e de controle (GC), que recebeu o tratamento após cinco semanas desta avaliação. Os registros eletromiográficos foram coletados na avaliação inicial (Av), imediatamente após uma sessão de acupuntura (R1) e ao final de dez sessões (R10) no GE. O GC foi avaliado no início (Av) e após cinco semanas (R10), enquanto aguardava o tratamento. A acupuntura foi realizada duas vezes na semana, por cinco semanas ininterruptas. Os resultados foram analisados estatisticamente. Resultados: Na posição de repouso mandibular, o GE apresentou redução significativa da atividade eletromiográfica do músculo temporal esquerdo em R1 e nos músculos temporal direito e esquerdo em R10. No GC, a atividade no músculo temporal esquerdo aumentou após cinco semanas. Na mastigação, houve redução da atividade no músculo masseter direito no GE. Conclusão: A acupuntura reduziu a atividade elétrica dos músculos temporais na posição de repouso mandibular, proporcionando melhor equilíbrio muscular entre estes e os músculos masseteres. Não houve um efeito uniforme da acupuntura sobre os músculos avaliados durante a máxima intercuspidação e a mastigação, não apresentando melhora na sua qualidade.
\end{abstract}

Descritores: Eletromiografia; Transtornos da articulação temporomandibular; Acupuntura; Músculos mastigatórios; Sistema estomatognático

\section{INTRODUÇÃO}

A desordem temporomandibular (DTM) caracteriza-se por dor na região da articulação temporomandibular, dor e/ou fadiga nos músculos craniocervicofaciais, especialmente os músculos mastigatórios, limitação e/ou desvios dos movimentos mandibulares, zumbido e presença de ruídos

Pesquisa realizada no Departamento de Fonoaudiologia, Laboratório de Motricidade Orofacial, Universidade Federal de Santa Maria - UFSM - Santa Maria (RS), Brasil.

(1) Programa de Pós Graduação (Mestrado) em Distúrbio da Comunicação Humana, Universidade Federal de Santa Maria - UFSM - Santa Maria (RS), Brasil.

(2) Departamento de Fisioterapia e Programa de Pós Graduação Distúrbio da Comunicação Humana, Universidade Federal de Santa Maria - UFSM Santa Maria (RS), Brasil.

(3) Departamento de Fonoaudiologia e Programa de Pós Graduação Distúrbio da Comunicação Humana, Universidade Federal de Santa Maria - UFSM Santa Maria (RS), Brasil.

Endereço para correspondência: Graciele da Silva Borin. Dr. Pantaleão, 377, Centro, Santa Maria (RS), Brasil, CEP: 97010-180. E-mail: graciborin@hotmail.com

Recebido em: 2/2/2011; Aceito em: 14/9/2011 articulares durante a função ${ }^{(1,2)}$. Sua etiologia envolve causas multifatoriais, podendo ser originada por associações entre fatores posturais, estruturais e psicológicos ${ }^{(3)}$.

A utilização da eletromiografia de superfície tem como objetivo auxiliar no diagnóstico e na terapêutica dos distúrbios motores orofaciais, nas alterações de respiração, mastigação, deglutição, fala e nas disfunções temporomandibulares. A utilização da eletromiografia de superfície tem sido importante dentro das terapias miofuncionais orofaciais por ser um método objetivo e quantitativo(4).

Atualmente, o tratamento da DTM inclui a educação do paciente, terapia comportamental, fisioterapia (ultrassom, eletro-estimulação transcutânea, laser, exercícios, massagem, mobilizações), acupuntura, Fonoaudiologia, placa miorrelaxante, ajustamento oclusal, cirurgia e intervenção medicamentosa ${ }^{(5,6)}$.

Nas últimas décadas, a acupuntura tornou-se cada vez mais popular e parcialmente aceita nos países ocidentais devido a seus efeitos positivos sobre o alívio da dor aguda e crônica $^{(7)}$. O uso da acupuntura em pacientes com DTM é considerado eficaz para o aumento da amplitude de movimento das ATMs, ativando a liberação de serotonina, encefalina e endorfina, pro- 
porcionando assim o relaxamento dos músculos mastigatórios e diminuição da dor ${ }^{(8)}$.

Em decorrência disto, vários autores citam a acupuntura como alternativa para tratamento da sintomatologia dolorosa da DTM $^{(9-12)}$.

Assim, este estudo propôs-se a avaliar o efeito da acupuntura na atividade eletromiográfica dos músculos mastigatórios (masseter e temporal anterior, bilateralmente) em pacientes com desordem temporomandibular (DTM).

\section{MÉTODOS}

Foram entrevistados 161 indivíduos, que foram avaliados e selecionados para participarem do estudo a partir dos seguintes critérios de inclusão: gênero feminino, idade entre 20 e 40 anos, relato de dor na região da articulação temporomandibular e músculos mastigatórios e diagnóstico de DTM segundo os Critérios de Diagnóstico para Pesquisa de Desordens Temporomandibulares (RDC-TMD) ${ }^{(13,14)}$ (Tabela 1). Foram excluídas do estudo voluntários que apresentassem falhas dentárias, doença reumatológica ou estivessem sob algum tratamento odontológico, fisioterapêutico ou medicamentoso.

Sendo assim, 52 indivíduos preencheram os critérios de inclusão para participarem do estudo. As 32 primeiras participantes foram submetidas à acupuntura duas vezes na semana por cinco semanas ininterruptas e foram reavaliadas a seguir. Apenas 20 participantes concluíram o tratamento e constituíram o grupo de estudo (GE). As demais voluntárias constituíram o grupo controle (GC); seus dados foram coletados no início e após cinco semanas sem tratamento, após as quais foram incluídas no tratamento.

Este estudo foi aprovado pelo comitê de Ética e Pesquisa da Universidade Federal de Santa Maria (23081.018541/2007). Todas as participantes assinaram o termo de consentimento livre e esclarecido.

\section{Procedimentos}

Foram coletados registros eletromiográficos dos músculos masseter e temporal (fascículo anterior), bilateralmente, nas posições de repouso, máxima intercuspidação e durante mastigação unilateral direita, mastigação unilateral esquerda e mastigação habitual ritmada. Para cada posição, foram realizadas três aquisições e, dentre estas, o sinal de melhor qualidade foi selecionado para a análise dos dados.

Durante as coletas, o indivíduo permaneceu sentado, em posição confortável, orientado a ficar com a coluna ereta, braços relaxados, pés apoiados no solo e o olhar fixo no horizonte. Para cada situação foram realizadas pelo menos três coletas com vistas à melhor qualidade possível do sinal eletromiográfico ${ }^{(15)}$. Primeiramente, foi coletado o sinal EMG na posição de repouso mandibular durante 20 segundos.

A máxima intercuspidação foi incentivada pelo examinador pelo comando verbal "aperta, aperta, aperta...". O sujeito realizava o máximo apertamento dentário, com material Parafilm ${ }^{\circledR}$ colocado entre os dentes pré-molares, primeiro e segundo molar inferior e superior, bilateralmente. $\mathrm{O}$ tempo de registro da contração foi de cinco segundos, com período de intervalo de dez segundos entre cada coleta, controlado pelo cronômetro $^{(16)}$.

Os registros da mastigação habitual ritmada, mastigação unilateral direita e unilateral esquerda foram obtidos em contrações concêntricas controladas por metrônomo digital da marca Cherub® - Tipo WSM 001A, ritmado em 60 bpm $^{(16)}$. O participante foi orientado inicialmente, a mastigar uma goma de mascar comercial por cinco segundos, para obter uma consistência uniforme antes da aquisição dos registros e, a seguir, este foi coletado durante dez segundos ${ }^{(17)}$.

O equipamento utilizado foi um eletromiógrafo EMG Lynx (Lynx Tecnologia Eletrônica Ltda) de oito canais com placa de conversão analógica-digital de 16 bits modelo CAD 10/26, frequência de amostragem de $2 \mathrm{kHz}$, filtro Butterworth, com frequência de corte passa-alta de $10 \mathrm{~Hz}$ e passa-baixa de 1 $\mathrm{kHz}$. A aquisição do sinal foi realizada por meio do programa BioInspector, desenvolvido pela Lynx Tecnologia Eletrônica Ltda. Os resultados do exame foram quantificados em RMS (raiz quadrada da média) pelo próprio programa de aquisição de dados, expressos em microvolts e armazenados em computador portátil sem conexão com a rede elétrica, a fim de evitar a interferência desta no sinal eletromiográfico.

Os eletrodos foram colocados no ventre muscular dos músculos masseteres bilaterais e temporais anteriores bilaterais, paralelamente à direção das fibras musculares. Sua localização foi definida com base na prova de função de cada músculo ${ }^{(16)}$. O eletrodo de referência foi colocado no punho direito do paciente visando evitar o efeito de interferência do ruído elétrico externo. Para facilitar a condução do potencial elétrico, a pele foi limpa e friccionada com algodão e álcool etílico $70 \%$ para a remoção das células mortas, antes dos eletrodos serem colocados.

Os resultados obtidos do sinal eletromiográfico foram processados em RMS (root mean square) e normalizados para melhor comparação entre os grupos. Este procedimento é recomendado quando as comparações são feitas entre sujeitos, dias, músculos ou diferentes estudos para reduzir a variabilidade introduzida pelos fatores intrínsecos e extrínsecos e possibilitar comparações. A normalização visa reduzir as diferenças entre registros de um mesmo sujeito, principalmente depois

Tabela 1. Frequência de diagnóstico de DTM nos diferentes subgrupos conforme o RDC/TMD - Eixo I do grupo de estudo e grupo controle

\begin{tabular}{llllccccc}
\hline \multirow{2}{*}{ Grupo } & & \multicolumn{2}{c}{ Subgrupos do RDC/TMD - Eixo I (\%) } & \multicolumn{2}{c}{ Sem } \\
\cline { 3 - 6 } & & I & I e III & III & I, II, III & I e II & diagnóstico \\
\hline GE & Av & 5 & 45 & 0 & 45 & 5 & 0 \\
GC & Av & 0 & 45 & 15 & 35 & 5 & 0 \\
\hline
\end{tabular}

Legenda: $\mathrm{GE}$ = grupo de estudo; GC = grupo controle; Diagnóstico I = desordem miofascial; Diagnóstico II = deslocamento de disco; Diagnóstico III = artralgia; $A v=$ avaliação inicial do grupo de estudo e grupo controle 
da retirada dos eletrodos, ou entre sujeitos diferentes, de forma a tornar a interpretação dos dados reprodutíveis. Assim, a normalização neste estudo foi realizada da seguinte forma: a mastigação unilateral direita e esquerda e a mastigação habitual pelo valor obtido no primeiro ciclo mastigatório, o repouso pela média dos valores obtidos do sinal da aquisição da máxima intercuspidação e a máxima intercuspidação pelo valor do seu pico máximo.

Para análise dos resultados, foram considerados os dados obtidos na avaliação inicial (Av), na reavaliação imediatamente após uma sessão de acupuntura (R1) e na reavaliação (R10) após dez sessões de acupuntura para o GE. Na R10 foram analisados os resultados eletromiográficos de 20 mulheres que completaram as dez sessões de acupuntura. Na R1 foram analisados os resultados da EMG de 38 participantes: $32 \mathrm{mu}-$ lheres que iniciaram o tratamento e não concluíram, realizando apenas uma sessão de acupuntura, e seis participantes do grupo controle que, após cinco semanas de espera pelo tratamento, realizaram apenas uma sessão de acupuntura. Para o GC foram analisados os resultados da avaliação inicial (Av) e após cinco semanas (R10), enquanto aguardavam o tratamento.

$\mathrm{O}$ tratamento de acupuntura foi realizado com agulhas descartáveis $(0,25 \times 0,15 \mathrm{~mm})$ inseridas nos respectivos pontos com a pele previamente limpa com algodão e álcool etílico $70 \%$. Durante a aplicação da terapia cada participante permaneceu deitado em uma maca, na posição de decúbito dorsal. A acupuntura foi realizada duas vezes na semana, durante 30 minutos, por cinco semanas ininterruptas, totalizando dez atendimentos. A aplicação da técnica de acupuntura foi realizada por uma das autoras e os pontos selecionados para o tratamento foram os referidos na literatura como pontos para o tratamento da DTM e pontos para ansiedade.

Assim, os pontos utilizados no tratamento foram os seguintes: ST7 (xiaguan: estômago 7: situa-se logo abaixo do arco zigomático e à frente da cabeça da mandíbula); ST5 (daying: situa-se na margem anterior do músculo masseter com a margem inferior do corpo da mandíbula); TE17 (yifeng: situa-se entre o processo mastóideo e o ramo da mandíbula); EXHN5 (tayang: situa-se cerca de 1,0 cun da depressão posterior ao ponto médio entre a extremidade lateral da sobrancelha e o canto externo do olho); GB43 (xiaxu: entre a cabeça do quarto e quinto metatarso), LI4 (hegu: entre o primeiro e segundo metacarpo) e EXHN3 (yntang: situa-se entre as extremidades médias das duas sobrancelhas) ${ }^{(12-18)}$ (Figuras 1 e 2).

A normalidade dos dados foi verificada pelo teste de Lilliefors. Os valores obtidos dos sinais eletromiográficos foram analisados pelo teste t de Student, sendo considerado um nível de significância de 5\% (p<0,05).

\section{RESULTADOS}

Os resultados eletromiográficos observados na R1, na posição de repouso mandibular, demonstraram diferença apenas para o músculo temporal esquerdo $(\mathrm{p}=0,0062)$ com diminuição do valor do potencial elétrico imediatamente após uma sessão de acupuntura. Os resultados obtidos na avaliação do sinal eletromiográfico do GE demonstraram uma atividade elétrica de repouso mandibular maior nos músculos temporais em relação

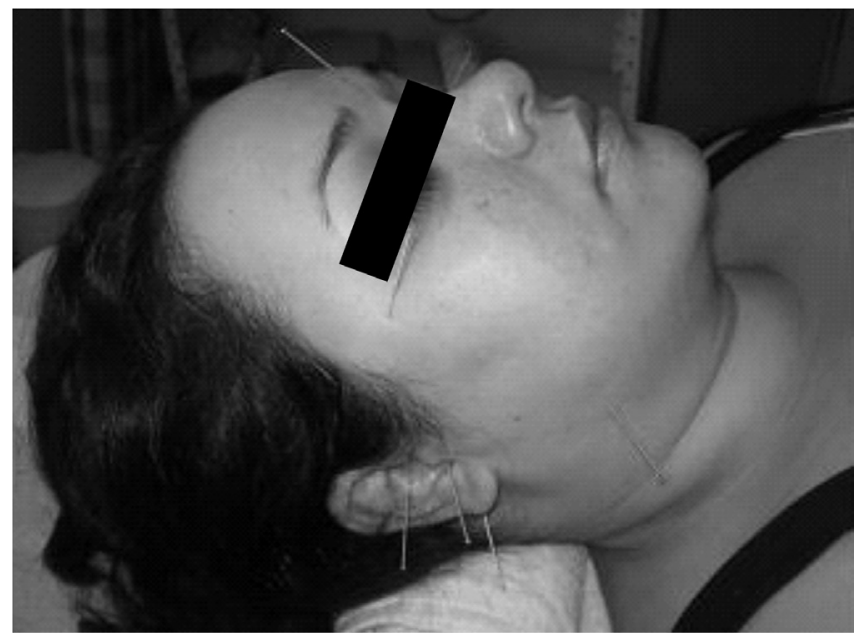

Figura 1. Pontos de acupuntura E7, E5, TA17, VB3, tayang e yntang

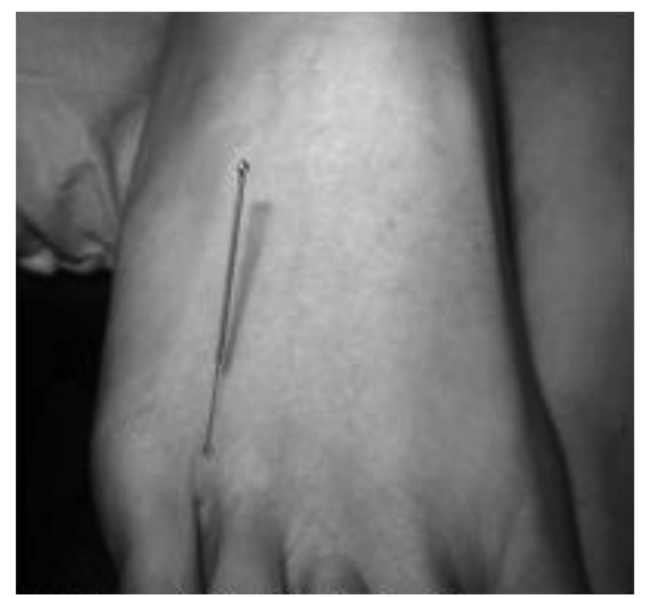

Figura 2. GB43 (xiaxu)

aos masseteres, com significância estatística, principalmente para o músculo temporal esquerdo. Após o tratamento houve diminuição dos valores eletromiográficos em repouso, com significância estatística nos músculos temporais. O GC apresentou maior atividade elétrica nos músculos temporais na avaliação e, na reavaliação, os músculos temporal esquerdo e masseter esquerdo apresentaram aumento da atividade elétrica, com prevalência do músculo temporal esquerdo. Não foi observada diferença na atividade elétrica dos músculos mastigatórios no GC e GE (R1 e R10) durante a máxima intercuspidação (Tabela 2). Na Tabela 3 demonstram-se os valores não normalizados.

$\mathrm{Na}$ mastigação habitual, o GE apresentou diferença no músculo masseter direito, com menores valores de potencial elétrico após o tratamento (R10). Tanto o GC como o GE (R10) não apresentaram diferença na mastigação habitual entre os valores obtidos na Av e R10 (Tabela 4).

Comparando-se os valores do GE na avaliação (Av) e reavaliação após acupuntura (R10), observou-se uma diminuição da atividade elétrica do músculo temporal esquerdo na mastigação unilateral esquerda. $\mathrm{O} \mathrm{GC}$ não apresentou diferença entre $\mathrm{Av}$ e R10, porém a atividade elétrica dos músculos temporais direito e esquerdo tiveram seus valores aumentados em R10. $\mathrm{Na}$ reavaliação imediatamente após a acupuntura (R1), não houve diferença na atividade eletromiográfica dos músculos avaliados (Tabela 4). 
Tabela 2. Valores normalizados (em porcentagem) do sinal EMG dos músculos mastigatórios no repouso mandibular e máxima intercuspidação

\begin{tabular}{|c|c|c|c|c|c|c|}
\hline Posição & & & Mas D & Mas E & TA D & TA E \\
\hline \multirow{9}{*}{ Repouso } & \multirow{6}{*}{ GE } & Av & $3,34+9,40$ & $2.35+3.00$ & $275+3.52$ & $3.51+5.79$ \\
\hline & & $\mathrm{R} 1$ & $1,68 \pm 0,89$ & $2,44 \pm 2,57$ & $2,56 \pm 2,61$ & $2,37 \pm 1,24$ \\
\hline & & Valor de $p$ & 0,427 & 0,527 & 0,943 & $0,0062^{*}$ \\
\hline & & $\mathrm{Av}$ & $1,82 \pm 1,33$ & $1,98 \pm 1,13$ & $2,26 \pm 1,18$ & $2,73 \pm 1,27$ \\
\hline & & $\mathrm{R} 10$ & $1,32 \pm 0,76$ & $1,62 \pm 1,03$ & $1,60 \pm 0,89$ & $2,22 \pm 2,08$ \\
\hline & & Valor de $p$ & 0,391 & 0,151 & $0,002^{*}$ & $0,009^{*}$ \\
\hline & \multirow{3}{*}{$\mathrm{GC}$} & Av & $1,62 \pm 1,43$ & $2,16 \pm 2,82$ & $2,73 \pm 2,86$ & $2,82 \pm 1,88$ \\
\hline & & $\mathrm{R} 10$ & $2,58 \pm 3,11$ & $3,59 \pm 5,7$ & $2,68 \pm 2,27$ & $3,59 \pm 1,91$ \\
\hline & & Valor de $p$ & 0,12 & $0,036^{*}$ & 0,374 & $0,016^{*}$ \\
\hline \multirow{9}{*}{ MI } & \multirow{6}{*}{ GE } & $A v$ & $69,73 \pm 15,57$ & $71,32 \pm 15,83$ & $72,9 \pm 13,59$ & $74,02 \pm 15,47$ \\
\hline & & $\mathrm{R} 1$ & $72,19 \pm 6,9$ & $72,56 \pm 7,31$ & $73,65 \pm 6,9$ & $75,41 \pm 11,12$ \\
\hline & & Valor de $p$ & 0,074 & 0,339 & 0,491 & 0,527 \\
\hline & & Av & $73,03 \pm 7,02$ & $73,70 \pm 5,59$ & $73,62 \pm 6,5$ & $78,82 \pm 6,74$ \\
\hline & & $\mathrm{R} 10$ & $70,58 \pm 11,31$ & $71,09 \pm 7,73$ & $75,49 \pm 6,6$ & $76,03 \pm 9,84$ \\
\hline & & Valor de $p$ & 0,306 & 0,166 & 0,391 & 0,238 \\
\hline & \multirow{3}{*}{$\mathrm{GC}$} & Av & $71,71 \pm 8,01$ & $74,14 \pm 12,48$ & $80,66 \pm 12,65$ & $81,11 \pm 17,24$ \\
\hline & & $\mathrm{R} 10$ & $70,36 \pm 14,02$ & $73,02 \pm 12,17$ & $78,00 \pm 10,38$ & $76,07 \pm 10,07$ \\
\hline & & Valor de $p$ & 0,648 & 0,650 & 0,395 & 0,313 \\
\hline
\end{tabular}

*Valores significativos $(\mathrm{p}<0,05)$ - Teste $t$ de Student

Legenda: Mas = masseter; $\mathrm{E}$ = esquerda; $\mathrm{D}=$ direita; $\mathrm{TA}$ = temporal anterior; $\mathrm{GC}$ = grupo controle; $\mathrm{GE}$ = grupo de estudo; $\mathrm{Av}=$ avaliação do grupo de estudo e grupo controle; R1 = reavaliação imediatamente após uma sessão de acupuntura; R10 = reavaliação do grupo controle após cinco semanas e grupo de estudo após dez sessões de acupuntura; $\mathrm{MI}$ = máxima intercuspidação; $\mathrm{DP}$ = desvio-padrão

Tabela 3. Valores não-normalizados (em porcentagem) do sinal EMG dos músculos mastigatórios no repouso mandibular e máxima intercuspidação

\begin{tabular}{|c|c|c|c|c|c|c|}
\hline Posição & & & $\begin{array}{c}\text { Mas D } \\
\text { Média } \pm \text { DP }\end{array}$ & $\begin{array}{c}\text { Mas E } \\
\text { Média } \pm \text { DP }\end{array}$ & $\begin{array}{c}\text { TA D } \\
\text { Média } \pm \text { DP }\end{array}$ & $\begin{array}{c}\text { TA E } \\
\text { Média } \pm \mathrm{DP}\end{array}$ \\
\hline \multirow{9}{*}{ Repouso } & \multirow{6}{*}{ GE } & $A v$ & $2,88 \pm 1,05$ & $2,95 \pm 1,14$ & $3,65 \pm 1,30$ & $3,99 \pm 1,22$ \\
\hline & & $\mathrm{R} 1$ & $2,48 \pm 0,77$ & $2,86 \pm 0,93$ & $3,25 \pm 1,23$ & $2,86 \pm 0,93$ \\
\hline & & Valor de $p$ & 0,086 & 0,393 & 0,094 & $0,00^{*}$ \\
\hline & & $\overline{A v}$ & $2,75 \pm 1,08$ & $2,88 \pm 1,00$ & $3,75 \pm 1,47$ & $4,00 \pm 1,07$ \\
\hline & & R10 & $2,59 \pm 0,87$ & $2,61 \pm 0,85$ & $2,78 \pm 0,72$ & $2,76 \pm 0,68$ \\
\hline & & Valor de $p$ & 0,595 & 0,236 & $0,026^{*}$ & $0,001^{*}$ \\
\hline & \multirow{3}{*}{ GC } & $A v$ & $2,71 \pm 1,73$ & $3,31 \pm 2,12$ & $3,80 \pm 2,97$ & $3,89 \pm 1,25$ \\
\hline & & $\mathrm{R} 10$ & $3,09 \pm 2,54$ & $3,27 \pm 4,1$ & $3,98 \pm 1,45$ & $4,52 \pm 2,27$ \\
\hline & & Valor de $p$ & $0,041^{*}$ & 0,254 & 0,090 & $0,027^{*}$ \\
\hline \multirow{9}{*}{ MI } & \multirow{6}{*}{ GE } & $A v$ & $227,30 \pm 162,68$ & $210,54 \pm 153,02$ & $213,53 \pm 132,23$ & $173,85 \pm 121,78$ \\
\hline & & $\mathrm{R} 1$ & $210,50 \pm 145,80$ & $202,26 \pm 155,91$ & $194,53 \pm 101,12$ & $164,80 \pm 98,70$ \\
\hline & & Valor de $p$ & $0,0271^{*}$ & 0,640 & 0,441 & 0,428 \\
\hline & & $\overline{A v}$ & $230,53 \pm 158,71$ & $222,78 \pm 175,53$ & $206,29 \pm 95,60$ & $186,72 \pm 83,19$ \\
\hline & & $\mathrm{R} 10$ & $250,45 \pm 179,32$ & $212,71 \pm 164,41$ & $219,41 \pm 128,83$ & $172,69 \pm 68,80$ \\
\hline & & Valor de $p$ & 0,156 & 0,940 & 0,411 & 0,654 \\
\hline & \multirow{3}{*}{$\mathrm{GC}$} & $A v$ & $244,02 \pm 140,89$ & $241,67 \pm 127,89$ & $204,51 \pm 135,67$ & $177,08 \pm 98,76$ \\
\hline & & $\mathrm{R} 10$ & $219,13 \pm 174,87$ & $198,05 \pm 102,76$ & $217,84 \pm 143,00$ & $161,86 \pm 134,56$ \\
\hline & & Valor de $p$ & $0,031^{*}$ & 0,530 & $0,021^{*}$ & 0,560 \\
\hline
\end{tabular}

*Valores significativos $(p<0,05)-$ Teste $t$ de Student

Legenda: Mas = masseter; $\mathrm{E}=$ esquerda; $\mathrm{D}=$ direita; $\mathrm{TA}=$ temporal anterior; $\mathrm{GC}=$ grupo controle; $\mathrm{GE}$ = grupo de estudo; $\mathrm{Av}=$ avaliação do grupo de estudo e grupo controle; R1 = reavaliação imediatamente após uma sessão de acupuntura; R10 = reavaliação do grupo controle após cinco semanas e grupo de estudo após dez sessões de acupuntura; $\mathrm{Ml}$ = máxima intercuspidação; DP = desvio-padrão

Os valores do potencial elétrico para mastigação unilateral direita não apresentaram diferença para o GC e GE em R1 e R10 (Tabela 4). Na Tabela 5 demonstram-se os valores não normalizados.

\section{DISCUSSÃO}

Os resultados obtidos pela análise do sinal eletromiográfico dos músculos mastigatórios do GE mostraram uma assincronia 
Tabela 4. Valores normalizados (em porcentagem) do sinal eletromiográfico dos músculos mastigatórios na mastigação habitual, mastigação unilateral esquerda e mastigação unilateral direita

\begin{tabular}{|c|c|c|c|c|c|c|}
\hline \multirow{2}{*}{ Mastigação } & & & Mas D & Mas E & TA D & TA E \\
\hline & & & Média \pm DP & Média \pm DP & Média \pm DP & Média \pm DP \\
\hline \multirow{9}{*}{$\mathrm{MH}$} & \multirow{6}{*}{ GE } & $A v$ & $31,90 \pm 20,96$ & $40,43 \pm 30,12$ & $46,33 \pm 25,27$ & $40,52 \pm 27,57$ \\
\hline & & $\mathrm{R} 1$ & $54,15 \pm 26,49$ & $42,44 \pm 28,08$ & $51,76 \pm 25,54$ & $44,68 \pm 24,89$ \\
\hline & & Valor de $p$ & 0,19 & 0,755 & 0,298 & 0,38 \\
\hline & & $A v$ & $119,24 \pm 47,87$ & $161,83 \pm 135,91$ & $148,07 \pm 132,21$ & $107,50 \pm 41,97$ \\
\hline & & $\underline{\mathrm{R} 10}$ & $102,65 \pm 25,68$ & $121,90 \pm 67,33$ & $105,21 \pm 29,30$ & $117,32 \pm 26,90$ \\
\hline & & Valor de $p$ & $0,045^{*}$ & 0,146 & 0,269 & 0,573 \\
\hline & \multirow{3}{*}{$\mathrm{GC}$} & $A v$ & $50,50 \pm 33,57$ & $48,35 \pm 33,10$ & $45,66 \pm 33,67$ & $50,59 \pm 33,09$ \\
\hline & & $\mathrm{R} 10$ & $48,60 \pm 36,86$ & $50,43 \pm 27,40$ & $51,05 \pm 37,11$ & $48,98 \pm 24,10$ \\
\hline & & Valor de $p$ & 0,789 & 0,754 & 0,691 & 0,765 \\
\hline \multirow{9}{*}{ MUE } & \multirow{6}{*}{ GE } & $A v$ & $39,93 \pm 38,49$ & $94,29 \pm 73,11$ & $49,94 \pm 51,94$ & $42,69 \pm 73,57$ \\
\hline & & $\mathrm{R} 1$ & $45,99 \pm 51,00$ & $94,56 \pm 68,85$ & $51,94 \pm 30,02$ & $69,70 \pm 38,72$ \\
\hline & & Valor de $p$ & 0,264 & 0,638 & 0,290 & 0,456 \\
\hline & & $\overline{A v}$ & $108,40 \pm 32,44$ & $112,77 \pm 27,96$ & $108,40 \pm 31,53$ & $121,28 \pm 53,27$ \\
\hline & & $\mathrm{R} 10$ & $132,29 \pm 86,07$ & $114,94 \pm 25,73$ & $111,27 \pm 65,10$ & $106,15 \pm 5,73$ \\
\hline & & Valor de $p$ & 0,209 & 0,815 & 0,892 & 0,298 \\
\hline & \multirow{3}{*}{$\mathrm{GC}$} & Av & $31,61 \pm 19,94$ & $66,51 \pm 41,38$ & $38,26 \pm 25,73$ & $52,00 \pm 32,13$ \\
\hline & & $\mathrm{R} 10$ & $35,21 \pm 22,25$ & $69,19 \pm 43,00$ & $44,00 \pm 24,49$ & $60,05 \pm 33,69$ \\
\hline & & Valor de $p$ & 0,176 & 0,215 & 0,244 & 0,239 \\
\hline \multirow{9}{*}{ MUD } & \multirow{6}{*}{ GE } & Av & $94,54 \pm 58,71$ & $94,29 \pm 73,11$ & $82,31 \pm 39,00$ & $73,57 \pm 42,69$ \\
\hline & & $\mathrm{R} 1$ & $84,11 \pm 59,17$ & $94,56 \pm 68,85$ & $74,03 \pm 33,88$ & $69,70 \pm 38,72$ \\
\hline & & Valor de $p$ & 0,61 & 0,337 & 0,57 & 0,29 \\
\hline & & $\overline{A v}$ & $117,17 \pm 10,58$ & $105,30 \pm 34,63$ & $116,78 \pm 34,55$ & $100,03 \pm 31,14$ \\
\hline & & $\mathrm{R} 10$ & $100,36 \pm 20,15$ & $100,30 \pm 32,80$ & $108,58 \pm 32,11$ & $91,83 \pm 24,84$ \\
\hline & & Valor de $p$ & 0,154 & 0,664 & 0,416 & 0,355 \\
\hline & \multirow{3}{*}{$\mathrm{GC}$} & $A v$ & $101,99 \pm 18,88$ & $101,93 \pm 20,18$ & $100,22 \pm 14,76$ & $97,33 \pm 15,15$ \\
\hline & & $\mathrm{R} 10$ & $102,52 \pm 28,57$ & $101,39 \pm 18,88$ & $121,36 \pm 73,38$ & $105,42 \pm 29,94$ \\
\hline & & Valor de $p$ & 0,948 & 0,941 & 0,240 & 0,202 \\
\hline
\end{tabular}

Teste t de Student. $(p<0,05)$.

Legenda: Mas = masseter; $\mathrm{E}=$ esquerda; $\mathrm{D}=$ direita; $\mathrm{TA}=$ temporal anterior; $\mathrm{GC}=$ grupo controle; $\mathrm{GE}$ = grupo de estudo; $\mathrm{MH}=$ mastigação habitual; $\mathrm{MUE}=$ mastigação unilateral esquerda; MUD = mastigação unilateral direita; $A v$ = avaliação do grupo de estudo e grupo controle; $R 1$ = reavaliação imediatamente após uma sessão de acupuntura; R10 = reavaliação do grupo controle após cinco semanas e grupo de estudo após dez sessões de acupuntura; DP = desvio-padrão

muscular, comum em pacientes com DTM(19). Em R1 do GE, houve diminuição significativa da atividade elétrica no músculo temporal esquerdo na posição de repouso mandibular. Com isso, a assincronia observada no lado esquerdo corrigiu-se após aplicação de acupuntura.

A melhor sincronia obtida do lado esquerdo após apenas uma aplicação de acupuntura é importante para a melhora da sintomatologia dos indivíduos com DTM porque, segundo alguns autores, o músculo temporal anterior, principalmente o esquerdo, é o que apresenta maior frequência de ativação com a mandíbula em posição de repouso, especialmente em pacientes que sofrem de cefaléia. A ativação dos músculos temporais também pode levar a mandíbula a uma posição retrusiva, produzindo dor na $\mathrm{ATM}^{(19)}$.

Após o tratamento com acupuntura (R10), observou-se diminuição do potencial elétrico em repouso dos músculos temporais esquerdo e direito, o que proporcionou melhor equilíbrio muscular entre os músculos masseteres e temporais, sugerindo melhora da DTM nos indivíduos deste estudo. Porém, os potenciais elétricos dos músculos temporais ainda apresentavam-se levemente mais elevados que nos masseteres. A hiperatividade dos músculos da mastigação corresponde a grande parte da etiologia das DTMs ${ }^{(20)}$. Nos indivíduos deste estudo houve diminuição do potencial elétrico dos músculos mastigatórios bilateralmente, o que indica menor contribuição deste fator etiológico na DTM.

Vários autores ${ }^{(21-23)}$ confirmam a maior atividade elétrica de repouso dos músculos mastigatórios, especialmente temporal anterior, em sujeitos com DTM, como encontrado neste estudo. Este comportamento é explicado pela necessidade de maior recrutamento muscular em pacientes com DTM e dor miofascial na situação de repouso mandibular ${ }^{(24,25)}$. Em nosso estudo ocorreu diminuição da dor e diminuição da atividade elétrica em repouso dos músculos temporais, indicando possível associação entre os fatores dor e aumento de atividade elétrica.

No presente estudo, após o tratamento com acupuntura, houve tendência a ao melhor equilíbrio entre os valores da atividade EMG dos músculos masseteres e temporais, porém a atividade dos músculos temporais ainda manteve o predomínio sobre os masseteres. Estes achados sugerem que o número de 
Tabela 5. Valores não-normalizados (em porcentagem) do sinal eletromiográfico dos músculos mastigatórios na mastigação habitual, mastigação unilateral esquerda e mastigação unilateral direita

\begin{tabular}{|c|c|c|c|c|c|c|}
\hline \multirow{2}{*}{ Mastigação } & & & Mas D & Mas E & TA D & TA E \\
\hline & & & Média \pm DP & Média \pm DP & Média \pm DP & Média \pm DP \\
\hline \multirow{9}{*}{$\mathrm{MH}$} & \multirow{6}{*}{ GE } & $A v$ & $31,86 \pm 21,82$ & $36,81 \pm 28,00$ & $43,95 \pm 24,62$ & $37,00 \pm 25,21$ \\
\hline & & $\mathrm{R} 1$ & $55,31 \pm 27,20$ & $38,67 \pm 25,27$ & $50,13 \pm 25,81$ & $41,83 \pm 23,42$ \\
\hline & & Valor de $p$ & 0,17 & 0,790 & 0,271 & 0,269 \\
\hline & & $\mathrm{Av}$ & $59,57 \pm 27,25$ & $57,04 \pm 71,98$ & $59,72 \pm 31,05$ & $51,27 \pm 40,60$ \\
\hline & & $\mathrm{R} 10$ & $45,65 \pm 36,64$ & $57,78 \pm 84,18$ & $42,22 \pm 30,23$ & $40,47 \pm 40,80$ \\
\hline & & Valor de $p$ & 0,149 & 0,753 & 0,066 & $0,028^{*}$ \\
\hline & \multirow{3}{*}{$\mathrm{GC}$} & $\mathrm{Av}$ & $50,50 \pm 33,57$ & $48,35 \pm 33,10$ & $45,66 \pm 33,67$ & $50,59 \pm 33,09$ \\
\hline & & $\mathrm{R} 10$ & $48,60 \pm 36,86$ & $50,43 \pm 27,40$ & $51,05 \pm 37,11$ & $48,98 \pm 24,10$ \\
\hline & & Valor de $p$ & 0,789 & 0,754 & 0,691 & 0,765 \\
\hline \multirow{9}{*}{ MUE } & \multirow{6}{*}{ GE } & $\mathrm{Av}$ & $39,20 \pm 39,13$ & $90,94 \pm 71,90$ & $47,68 \pm 29,57$ & $72,23 \pm 42,75$ \\
\hline & & $\mathrm{R} 1$ & $45,70 \pm 51,84$ & $90,64 \pm 66,52$ & $50,44 \pm 29,33$ & $68,76 \pm 39,02$ \\
\hline & & Valor de $p$ & 0,734 & 0,428 & 0,393 & 0,382 \\
\hline & & $A v$ & $106,97 \pm 66,82$ & $44,98 \pm 39,93$ & $87,52 \pm 40,01$ & $46,77 \pm 20,48$ \\
\hline & & R10 & $41,41 \pm 32,18$ & $106,55 \pm 72,18$ & $51,15 \pm 30,47$ & $73,92 \pm 444,22$ \\
\hline & & Valor de $p$ & $0,00^{*}$ & $0,003^{*}$ & $0,002^{*}$ & $0,005^{\star}$ \\
\hline & \multirow{3}{*}{$\mathrm{GC}$} & $\mathrm{Av}$ & $31,61 \pm 19,94$ & $66,51 \pm 41,38$ & $38,26 \pm 25,73$ & $52,00 \pm 32,13$ \\
\hline & & $\mathrm{R} 10$ & $35,21 \pm 22,25$ & $69,19 \pm 43,00$ & $44,00 \pm 24,49$ & $60,05 \pm 33,69$ \\
\hline & & Valor de $p$ & 0,176 & 0,215 & 0,244 & 0,239 \\
\hline \multirow{9}{*}{ MUD } & \multirow{6}{*}{ GE } & $A v$ & $94,90 \pm 59,68$ & $40,49 \pm 87,33$ & $80,70 \pm 38,61$ & $43,55 \pm 20,87$ \\
\hline & & $\mathrm{R} 1$ & $83,93 \pm 60,17$ & $45,40 \pm 44,67$ & $72,84 \pm 33,73$ & $41,12 \pm 24,71$ \\
\hline & & Valor de $p$ & 0,789 & 0,587 & 0,753 & 0,645 \\
\hline & & $A v$ & $227,98 \pm 162,02$ & $218,61 \pm 179,64$ & $200,87 \pm 103,45$ & $180,85 \pm 91,11$ \\
\hline & & $\mathrm{R} 10$ & $116,93 \pm 67,00$ & $47,12 \pm 36,44$ & $90,21 \pm 43,14$ & $49,26 \pm 29,40$ \\
\hline & & Valor de $p$ & $0,001^{*}$ & $0,000^{*}$ & $0,00^{*}$ & $0,00^{*}$ \\
\hline & \multirow{3}{*}{$\mathrm{GC}$} & $A v$ & $70,33 \pm 52,82$ & $43,02 \pm 34,19$ & $75,22 \pm 58,37$ & $50,14 \pm 39,91$ \\
\hline & & $\mathrm{R} 10$ & $74,73 \pm 48,97$ & $36,32 \pm 26,85$ & $79,09 \pm 45,96$ & $43,96 \pm 33,53$ \\
\hline & & Valor de $p$ & 0,703 & 0,249 & 0,874 & 0,654 \\
\hline
\end{tabular}

*Valores significativos $(p<0,05)-$ Teste $t$ de Student

Legenda: Mas = masseter; $\mathrm{E}=$ esquerda; $\mathrm{D}=$ direita; $\mathrm{TA}=$ temporal anterior; $\mathrm{GC}=$ grupo controle; $\mathrm{GE}=$ grupo de estudo; $\mathrm{MH}=$ mastigação habitual; $\mathrm{MUE}=$ mastigação unilateral esquerda; MUD = mastigação unilateral direita; $A v$ = avaliação do grupo de estudo e grupo controle; R1 = reavaliação imediatamente após uma sessão de acupuntura; R10 = reavaliação do grupo controle após cinco semanas e grupo de estudo após dez sessões de acupuntura; DP = desvio-padrão

sessões de acupuntura pode ter sido insuficiente e haja necessidade da associação com outras modalidades terapêuticas.

No GC havia maior atividade elétrica de repouso nos músculos temporais em relação aos masseteres em Av; em R10, esta atividade aumentou significativamente nos músculos temporal e masseter esquerdos. Tais achados evidenciaram piora da DTM neste grupo após o período de cinco semanas, enquanto aguardavam pelo tratamento.

Neste estudo, apesar dos valores de atividade eletromiográfica aumentados nos músculos temporais, estes não atingiram níveis de hiperatividade muscular em ambos os grupos. Valores de repouso mandibular acima de $10 \%$ da contração voluntária máxima, ou seja, máxima intercuspidação, são considerados como hiperatividade ${ }^{(25)}$. Corroborando nossos achados, um estudo $^{(26)}$ verificou maior atividade elétrica de repouso dos músculos mastigatórios de indivíduos com DTM em relação a controles. Porém, os valores de atividade não atingiram níveis de hiperatividade muscular e foi considerado um sinal sugestivo de DTM.

Resultados variáveis quanto ao efeito da acupuntura foram encontrados na máxima intercuspidação. Ao contrário de nosso estudo, autores ${ }^{(27)}$ que avaliaram pacientes com DTM por meio da EMG após dez sessões de acupuntura observaram menor atividade durante o apertamento dental e diminuição da sintomatologia dolorosa após o tratamento. Os autores explicam que estes pacientes despendem maior esforço para efetuar os movimentos de força, provavelmente pelo desequilíbrio muscular, que dificulta a função normal. Também um estudo ${ }^{(26)}$ que investigou o efeito da TENS ${ }^{\circledR}$ demonstrou diminuição da dor e da atividade eletromiográfica dos músculos temporais e aumento da atividade dos masseteres durante a máxima intercuspidação em pacientes com DTM miogênica.

Em concordância com nosso estudo, outros ${ }^{(28)}$ relataram que distúrbios da articulação temporomandibular podem influenciar negativamente o comportamento de mastigação e verificaram que, após o tratamento com aparelho oclusal e fisioterapia (programa de auto-apoio) o desempenho dos músculos mastigatórios, avaliado através de EMG, manteve-se inalterado e a dor, assim como a força de mordida e a duração do ciclo, apresentaram melhora significativa. 
Por outro lado, um estudo ${ }^{(27)}$ que avaliou indivíduos com DTM, por meio da EMG, demonstrou um aumento da atividade elétrica dos músculos masseteres direito e temporal direito durante atividades mastigatórias habituais de amendoins e uva passas após dez sessões terapia de acupuntura, e diminuição da atividade EMG nos músculos temporal e masseteres esquerdo após este tratamento. A ativação eletromiográfica aumentada nos músculos do lado direito após a acupuntura pode ter sido ocasionada pela mastigação preferencial dos alimentos deste lado, pois sabe-se que no lado de trabalho a atividade eletromigráfica é maior. Os autores ainda avaliaram a força bilateral com um dinamômetro adaptado, a qual aumentou após o tratamento, demonstrando que a acupuntura leva a uma diminuição da dor que contribui para o aumento da força.

Corroborando os resultados de nosso estudo, outros autores ${ }^{(19)}$ observaram, em indivíduos com DTM, maiores potenciais de ação durante a mastigação nos músculos temporais que nos masseteres, que são músculos de potência mastigatória e deveriam ser mais recrutados nesta atividade. Assim, os músculos temporais deixam sua principal função de posicionadores do côndilo, tornando-se hiperativos na função de mastigação, enquanto os masseteres podem ser considerados hipofuncionantes. Em nosso estudo, na avaliação do GE havia uma importante assincronia entre masseteres e temporais que diminuiu após a terapia com acupuntura (R10), proporcionando maior equilíbrio entre os potenciais elétricos desses músculos.

Há algumas evidências de que os seguintes tratamentos podem ser eficazes em aliviar a dor da DTM: aparelhos oclusais, acupuntura, terapia comportamental, exercícios mandibulares, treinamento postural e farmacoterapia. Evidências para o efeito das eletroterapias e a cirurgia são insuficientes e o ajuste oclusal parece não ter efeito ${ }^{(13,29)}$.
Alguns estudos ${ }^{(13,29)}$ encontraram evidências de que a acupuntura é melhor que nenhum tratamento e é melhor comparável a outras formas de tratamento conservadores. Isto foi confirmado em nosso estudo, pois o GC não apresentou melhora, mas sim piora, com aumento da atividade elétrica em repouso do músculo temporal esquerdo. No entanto, devido às deficiências metodológicas, pesquisadores ${ }^{(29)}$ recomendam $^{2}$ diretrizes específicas para melhorar a qualidade desta intervenção e, antes de a eficácia da acupuntura ser determinada, mais estudos primários são necessários.

Os achados referentes à assincronia entre os músculos masseteres e temporais nas situações de máxima intercuspidação e mastigação sugerem uma limitação da acupuntura como método terapêutico único e demonstram a necessidade da associação de outras modalidades terapêuticas, principalmente a terapia orofacial, pois além de ajudar na assincronia encontrada nos resultados poderá ajudar na manutenção dos bons resultados para pacientes com DTM.

\section{CONCLUSÃO}

A acupuntura reduziu a atividade elétrica na posição de repouso mandibular dos músculos temporais, o que proporcionou melhor equilíbrio entre os músculos masseteres e temporais.

A ação da acupuntura não ocorreu de forma uniforme sobre os músculos mastigatórios nas situações de mastigação e da máxima intercuspidação, não apresentando melhora na qualidade destas.

Os resultados positivos obtidos com a acupuntura podem ser atribuídos à sua abordagem com enfoque tanto nos aspectos físicos como emocionais presentes na DTM. Este método é de baixo custo, rápida aplicação, não apresenta efeitos colaterais e ainda pode ser utilizado como coadjuvante com outras terapias.

\begin{abstract}
Purpose: To assess the effect of acupuncture on the electromyographic activity of masticatory muscles in patients with temporomandibular disorder (TMD). Methods: Forty women, from 20 to 40 years old, with TMD diagnosis were assessed using the electromyography of the masseter and temporal (anterior fascicle) muscles. Participants were distributed into study group (SG), which received acupuncture treatment soon after the first assessment, and control group (CG), which received the treatment five weeks after this assessment. The electromyography was accomplished at the first evaluation (Av), immediately after one session of acupuncture (R1), and after ten sessions (R10) in the SG. The CG was evaluated at the beginning (Av) and after five weeks (R10), while waiting for the treatment. The acupuncture was carried out twice a week, for five uninterrupted weeks. Results were statistically analyzed. Results: At the rest mandibular position, the SG showed a significant decrease in the left temporal muscle activity in R1, and in the right and left temporal muscles in R10. In the CG, the activity increased in the left temporal muscle after five weeks (R10). During chewing, the activity decreased in the right masseter in the SG. Conclusion: Acupuncture reduced the temporal muscles activity at rest mandibular position, providing better muscular balance between these and masseter muscles. There was not a uniform effect of the acupuncture on the assessed muscles assessed during maximal intercuspal position and chewing, showing no improvement in the quality of their activity.
\end{abstract}

Keywords: Electromyography; Temporomandibular joint disorders; Acupuncture; Masticatory muscles; Stomatognathic system 


\section{REFERÊNCIAS}

1. Bonjardim LR, Gavião MB, Pereira LJ, Castelo PM, Garcia RC. Signs and simptoms of temporomandibular disorders in adolescents. Braz Oral Res. 2005;19(2):235-9.

2. Munhoz WC, Marques AP, de Siqueira JT. Evaluation of body posture in individuals with internal temporomandibular joint derangement. Cranio. 2005;23(4):269-77.

3. Suvinen TI, Kemppainen P. Review of clinical EMG studies related to muscle and occlusal factors in healthy and TMD subjects. J Oral Rehabil. 2007;34(4):631-44.

4. Rahal A, Pierotti S. Eletromiografia e cefalometria na fonoaudiologia. In: Ferreira LP, Befi-Lopes DM, Limongi SC, organizadores. Tratado de fonoudiologia. São Paulo: Roca; 2004. p.237-53.

5. Goudot P, Jaquinet AR, Hugonnet S, Haefliger W, Richter M. Improvement of pain and function after arthroscopy and arthrocentesis of the temporomandibular joint: a comparative study. J Craniomaxillofac Surg. 2000;28(1):39-43.

6. Cho SH, Whang WW. Acupuncture for temporomandibular disorders: a systematic review. J Orofac Pain. 2010;24(2):156-62.

7. Wong T. Use of electrostimulation of acupuncture points in general dental practice. Anesth Prog. 2001;36(4-5):243-4.

8. Lee MH, Liao SJ. Acupuntura em fisiatria. In: Kottle FJ, Lehmann JF. Tratado de medicina física e reabilitação de Krusen. $4^{\mathrm{a}}$ ed. Vol.1. São Paulo: Manole; 1994. p.

9. Smith P, Mosscrop D, Davies S, Sloan P, Al-Ani Z. The efficacy of acupuncture in the treatment of temporomandibular joint myofascial pain: a randomised controlled trial. J Dent. 2007;35(3):259-67.

10. Shin BC, Ha CH, Song YS, Lee MS. Effectiveness of combining manual therapy and acupuncture on temporomandibular joint dysfunction: a retrospective study. Am J Chin Med. 2007;35(2):203-8.

11. Okeson JP. Dor orofacial: guia de avaliação, diagnóstico e tratamento. 6a ed. São Paulo (SP): Quintessence; 2008.

12. Dallanora LJ, Faltin PP, Inoue RT, Santos VM. Avaliação do uso de acupuntura no tratamento de pacientes com bruxismo. RGO (Porto Alegre). 2004;52(5)333-9.

13. List T, Axelsson T. Management of TMD: evidence from systematic reviews and meta-analyses. J Oral Rehabil. 2010;37(6):430- 51.

14. De Felício CM, Sidequersky FV, Tartaglia GM, Sforza C. Electromyographic standardized indices in healthy Brazilian young adults and data reproducibility. J Oral Rehabil. 2009;36(8);577-83.

15. De Luca C. The use of surface electromyography in biomechanics. J Appl Biomech. 1997;13(2):135-63.

16. Borini $\mathrm{CB}$. Análise da atividade eletromiográfica de músculos mastigatórios em portadores de DTM durante mastigação [Dissertação]. Piracicaba: Universidade Estadual de Campinas; 2005.
17. Hanawa S, Tsuboi A, Watanabe M, Sasaki K. EMG study for perioral facial muscles function during mastication. J Oral Rehabil. 2008;35(3):159-70.

18. Rosted P, Bundgaard M, Pedersen AM. The use of acupuncture in the treatment of temporomandibular dysfunction - an audit. Acupunct Med. 2006; 24(1):16-22.

19. Bérzin F, Sakai E. Fundamentos da eletromiografia (EMG): da teoria à técnica. In: Fiúza SC, Martins NS, Dominguez-Rodrigues GC, Grimberg J, Pereira CB, Corrêa J, et al, organizadores. Nova visão em ortodontia e ortopedia funcional dos maxilares. São Paulo: Ed Santos; 2004. p.31130.

20. Yi LC, Guedes ZC, Vieira MM. Relação da postura corporal com a disfunção da articulação temporomandibular: hiperatividade dos músculos da mastigação. Fisioter Bras. 2003;4(5):341-7.

21. Pedroni CR, De Oliveira AS, Guaratini MI. Prevalence study of signs and symptoms of temporomandibular disorders in university students. J Oral Rehabil. 2003;30(3):283-9.

22. Castroflorio T, Icardi K, Becchino B, Merlo E, Debernardi C, Bracco $\mathrm{P}$, et al. Reproducibility of surface EMG variables in isometric submaximal contractions of jaw elevator muscles. J Electromyogr Kinesiol. 2006;16(3):498-505.

23. Boderé C, Téa SH, Giroux-Metges MA, Woda A. Activity of masticatory muscles in subjects with different orofacial pain conditions. Pain. 2005;116(1-2):33-41.

24. Venezian GC, da Silva MA, Mazzetto RG, Mazzetto MO. Low level laser effects on pain to palpation and electromyographic activity in TMD patients: a double-blind, randomized, placebo-controlled study. Cranio. 2010;28(2):84-91.

25. Finsterer JF. EMG-interference pattern analysis. J Electromyogr Kinesiol. 2001;11(4):231-46.

26. Rodrigues D, Siriani AO, Bérzin F. Effect of convencional TENS on pain and electromyographic activity of masticatory muscles in TMD patients. Braz Oral Res. 2004;18(4):290-5.

27. Rancan SV, Bataglion C, Bataglion SA, Bechara OM, Semprini M, Siéssere $\mathrm{S}$, et al. Acupuncture and temporomandibular disorders: a 3-month follow-up EMG study. J Altern Complement Med. 2009;15(12):1307-10.

28. Pereira Júnior FJ, Favilla EE, Dworkin S, Huggins K. Critérios de diagnóstico para pesquisa das disfunções temporomandibulares (RDC/ TMD). Tradução oficial para a língua portuguesa. JBC J Bras Clin Odontol Integr. 2004:8(47):384-95.

29. MacPherson H, White A, Cummings M, Jobst KA, Rose K, Niemtzow $\mathrm{RC}$, et al. Standards for Reporting Interventions in Controlled Trials of Acupuncture: the STRICTA recommendations. J Altern Complement Med. 2002;8(1):85-9. 